\title{
SURFACE POLISHING POSITIVELY INFLUENCES EASE OF PLATE AND SCREW REMOVAL
}

\author{
J.S. Hayes ${ }^{1,2}$, U. Seidenglanz ${ }^{1}$, A.I. Pearce ${ }^{1,2}$, S.G. Pearce ${ }^{1}$, C.W. Archer ${ }^{2}$ and R.G. Richards ${ }^{1,2}$ \\ 1.AO Research Institute Davos, Switzerland \\ 2.Cardiff School of Biosciences, Cardiff University, Wales, U.K.
}

\begin{abstract}
Difficulties removing temporary fracture fixation devices due to excessive bony on-growth results in extended surgical time leading to excessive blood loss, debris contamination and potentially refracture. Commercially available locking plates and screws are manufactured for clinics with a micro-rough surface, which contributes to the excessive bony on-growth reported. We have applied polishing technology to commercially pure titanium locking compression plates (LCP) and titanium-6\%aluminium$7 \%$ niobium (TAN) plates and screws to assess if it can alleviate problems with strong bony overgrowth. Samples were implanted for 6,12 and 18 months in a bilateral sheep tibia non fracture model and assessed for screw removal torque, percentage of bone contact and tissue-material response. Both electropolishing $(p=0.001)$ and paste polishing $(p=0.010)$ of TAN screws significantly reduced the mean torque required for removal compared to their micro-rough counterparts. This was accompanied by a trend for a lower percentage of bone contact for polished screws. This difference in bone contact was significant for paste polished TAN screws $(p<0.001)$ but not electropolished TAN screws $(p=0.066)$. Ex vivo, soft tissue removal was much easier ( five minutes) for polished constructs, which was difficult and at least four times longer for standard micro-rough constructs. We suggest that polishing of locked plate/screw systems will improve ease of removal and reduce implant related removal complications encountered due to excessive strong bony on-growth while maintaining biocompatibility and implant stability. Future studies aim to assess the potential of this technology in the next level of complication, a fracture model.
\end{abstract}

Keywords: Implant removal, in vivo, locking compression plate, screw removal, commercially pure titanium, titanium6\%aluminium-7\%niobium.

\footnotetext{
*Address for correspondence:

R.G. Richards

AO Research Institute Davos

AO Foundation

Clavadelerstrasse, 8

CH-7270 Davos Platz

Switzerland.
}

Telephone Number: +41 (0)81 4142441

E-mail: geoff.richards@aofoundation.org

\section{Introduction}

A recent survey suggests that approximately $42 \%$ of orthopedic surgeons routinely remove internal fixation devices (Hanson et al., 2008). In the United States, approximately $5 \%$ of planned orthopedic procedures involve removal of internal fixators (IF; Busam et al., 2006) while in Finland approximately $81 \%$ of implants are removed which accounts for $30 \%$ of all planned orthopedic procedures (Bostman and Pilhajamaki, 1996). Despite the 'routine' nature of device removal controversy still exists with many surgeons advocating implant retention over removal mainly due to implant removal related morbidity, or what is deemed to be a lack of scientific evidence in support of removal (Busam et al., 2006).

Studies have shown that subsequent to fulfilling its function a device can negatively affect the host giving rise to complications such as delayed infections (Highland and LaMont, 1985; Emel et al., 2007), allergic reactions (Summer et al., 2007), implant loosening or breakage (Sommer et al., 2005), device migration (Seipel et al., 2001) and hindrance of skeletal growth (Togrul et al., 2005). For hand applications the irritation of overlying free gliding tendons in contact with the plate is also of concern (Nazzal et al., 2006). Furthermore, the negative influence of metal ions and salts on adjacent tissues and organs is well documented (Case et al., 1994: Kasai et al., 2003: Keegan et al., 2007; Summer et al., 2007). Device extraction in children can further cloud the matter (Rosson and Shearer, 1991; Kahle, 1994; Alzahrani et al., 2003; Kim et al., 2005; Peterson, 2005; Busam et al., 2006). Removal is also advocated in fracture non-unions; when an implant protrudes into a joint; causing pain or when exposed in the oral cavity (Mosbah et al., 2003; Bhatt et al., 2005; Rallis et al., 2006).

However, implant removal is not without complication. One of the principle contributors to implant removal morbidity is difficulty in removing the device due to excessive bony on-growth. Recently in an AO symposium dedicated to implant removal problems (Salzburg, April 2008) a consensus of 150 surgeon participants (from Germany, Switzerland, and Austria) suggest that the removal of IFs can have complications of up to $40 \%$. Clinical observations have noted bone growth to encase the implant as well as growing up within the screw holes (Georgiadis et al., 2004). The unwanted presence of direct bone on-growth in these areas increases surgical time and demand as well as increasing the risk to the patient by augmenting blood loss and exposure to anesthesia (Swiontkowski, 1983; Alzahrani et al., 2003; Ilchmann and Parsch, 2006) as well as resulting in iatrogenic factors such as screw stripping, implant breakage and debris contamination. 
While excessive bony on-growth is reported for intramedullary nails and plate systems the problem appears to be extreme with locking plates given they are more stable than compression plating systems (Babst et al., 2003). Currently commercially pure titanium (cpTi) locking compression plates and titanium-6\%aluminium$7 \%$ niobium (TAN) screws (as well as all other IFs with the exception of hand polished hand plates) are provided for clinics with a 'standard' micro-rough surface. However, the ability of 'standard' cpTi and TAN to promote direct bone on-growth has been implicated as the cause of removal difficulties (Qu et al., 1996; Ferris et al., 1999; Boyan et al., 2002; Pearce et al., 2008; Hayes et al., 2009a). Therefore, in this in vivo sheep non-fracture model study we hypothesize that a smooth surface created by polishing of standard micro-rough cpTi LCPs and TAN screws will help to eliminate the removal problems associated with excessive direct bone-on-growth.

\section{Materials and Methods}

Implants were provided by SYNTHES ${ }^{\circledR}$. A 4-hole locking compression plate (LCP; $51 \mathrm{~mm}$ in length, $3.5 \mathrm{~mm}$ diameter) with self-tapping, locking head screws (12mm) were used. Materials included electropolished stainless steel (SS) plates with SS screws as a control system; 'standard' micro-rough commercially pure titanium (cpTiS) plate with standard micro-rough titanium$6 \%$ aluminium-7\%niobium (TAN-S) screws as a control for osseointegration; and two experimental systems consisting of paste polished cpTi (cpTi-PP) plates with paste polished TAN (TAN-PP) screws; and electropolished cpTi (cpTi-EP) plates with electropolished TAN (TANEP) screws. After preparation of standard micro-rough samples a subset of the screws and plates were ground, cleaned and either electro- or paste polished. The electropolished surfaces were produced by immersing the samples in a liquid electrolyte and applying an electric current (Steiger SA, Vionnaz, Switzerland). Mechanically paste polished samples were polished by KKS (KKS Ultraschall AG, Steinen, Switzerland) by tumbling with ground bark. All cpTi plates and TAN screws were anodized as the final finishing process with the exception of the cpTi-EP plates with TAN-EP screws. Prior to implantation implants were packaged individually and steam sterilized at $134^{\circ} \mathrm{C}$ for 20 minutes.

\section{Surface characterisation}

The surface topography of each material was quantitatively measured with a non-contact white-light FRT MicroProf 200 Profilometer (Fries Research \& Technology, Bergisch Gladbach, Germany). Roughness average (Ra- arithmetic mean of the absolute values of all points of the profile) of three separate implants ( 2 measurements per implant) were measured from a $0.5 \times 0.5 \mathrm{~mm}$ analysis area scan at a point density of 1000 points $/ \mathrm{mm}$.

Contact angle measurements for the LCPs were made using the Sessil drop method with the Drop Shape Analysis System (Contact Angle Measuring Instrument G10 and
DSA 10 Control Unit, Krüss GmbH, Hamburg, Germany) and analyzed using the Drop Shape Analysis 1.50 software (Krüss $\mathrm{GmbH}$ ). Contact angle was not preformed for the screws due to their small size and hence difficulty in collecting an accurate measurement.

The morphology of the plate and screw surfaces was examined using a Hitachi S4700 scanning electron microscope (SEM). Images were taken in the secondary electron mode with an accelerating voltage of $5 \mathrm{kV}$.

The chemical composition of the surfaces was assessed using X-ray photoelectron spectroscopy (XPS). All spectra were recorded on a Kratos Axis Nova (Kratos Analytical, Manchester, UK) using monochromatic $\mathrm{AlK} \alpha$ radiation $(1486.69 \mathrm{eV})$ produced at an anode power of $225 \mathrm{~W}(15 \mathrm{kV}$, $15 \mathrm{~mA}$ ), an electron take-off angle of $90^{\circ}$ relative to the surface plane and an electron analyzer pass energy of 80 $\mathrm{eV}$. During analysis the base pressure remained below $10^{-8}$ Torr. For quantification survey scans with a step width of $0.5 \mathrm{eV}$ were performed on two spots of $300 \times 700 \mu \mathrm{m}^{2}$ per sample. Data was evaluated with CasaXPS 2.3.10 (www.casaxps.com) using relative sensitivity factors supplied with the instrument.

\section{Surgical procedure}

Approval to perform this study was granted by the Cantonal Animal Ethics Committee (GR \#6/2006). Eighteen Swiss Alpine sheep were selected from an orthopedic research flock such that size, shape and age were standardized. A bilateral model was used consisting of two test constructs implanted on each tibia, (2 plates approximately $1 \mathrm{~cm}$ apart on each tibia). Therefore each animal received each treatment implant. Location of the implant was randomized. Sheep were separated into 3 groups namely 6, 12 and 18 months.

Animals were anesthetized (Diazepam (Valium $\left.{ }^{\circledR}\right) 0.3$ $\mathrm{mg} / \mathrm{kg}$ i.v. and Butorphanol (Morphasol ${ }^{\circledR}$ ) $0.08 \mathrm{mg} / \mathrm{kg}$ i.v.; Thiopenthal (Penthotal ${ }^{\circledR}$ ) 4-8 mg/kg i.v.; Isoflurane (approx $2 \%$ in oxygen: Oxygen flow rate was approx 0.5 $\mathrm{L} / \mathrm{min}$ )) and placed in lateral recumbency. Both tibiae were aseptically prepared for surgery. A $12 \mathrm{~cm}$ skin incision was made over the medial aspect of the tibia and continued down to the bone ensuring that the periosteum remained in situ and was not damaged. Screw holes were drilled using a $2.7 \mathrm{~mm}$ drill bit with saline irrigation to prevent thermal necrosis. Placement of the locking-head screws was done using a $1.5 \mathrm{Nm}$ torque limiting screwdriver. The distal plate was applied first such that the most distal screw hole was positioned $4 \mathrm{~cm}$ proximal to the medial malleolus of the tibia. The most proximal and distal screws in each plate were inserted first followed by the two central screws to ensure central placement of the plate on the tibia. The subcutaneous tissues were closed with resorbable sutures and the skin closed with stainless steel skin staples (removed 10-14 days post operatively). A light adhesive bandage was placed over the medial aspect of each tibia to protect the wound from contamination during the recovery phase. Sheep were radiographed every 4 weeks for the duration of the study. The final radiographs were taken after euthanasia. Postoperative analgesia consisted of Caprofen (Rimadyl®; 4 mg/kg, i.m., q24h) for 5 days, 


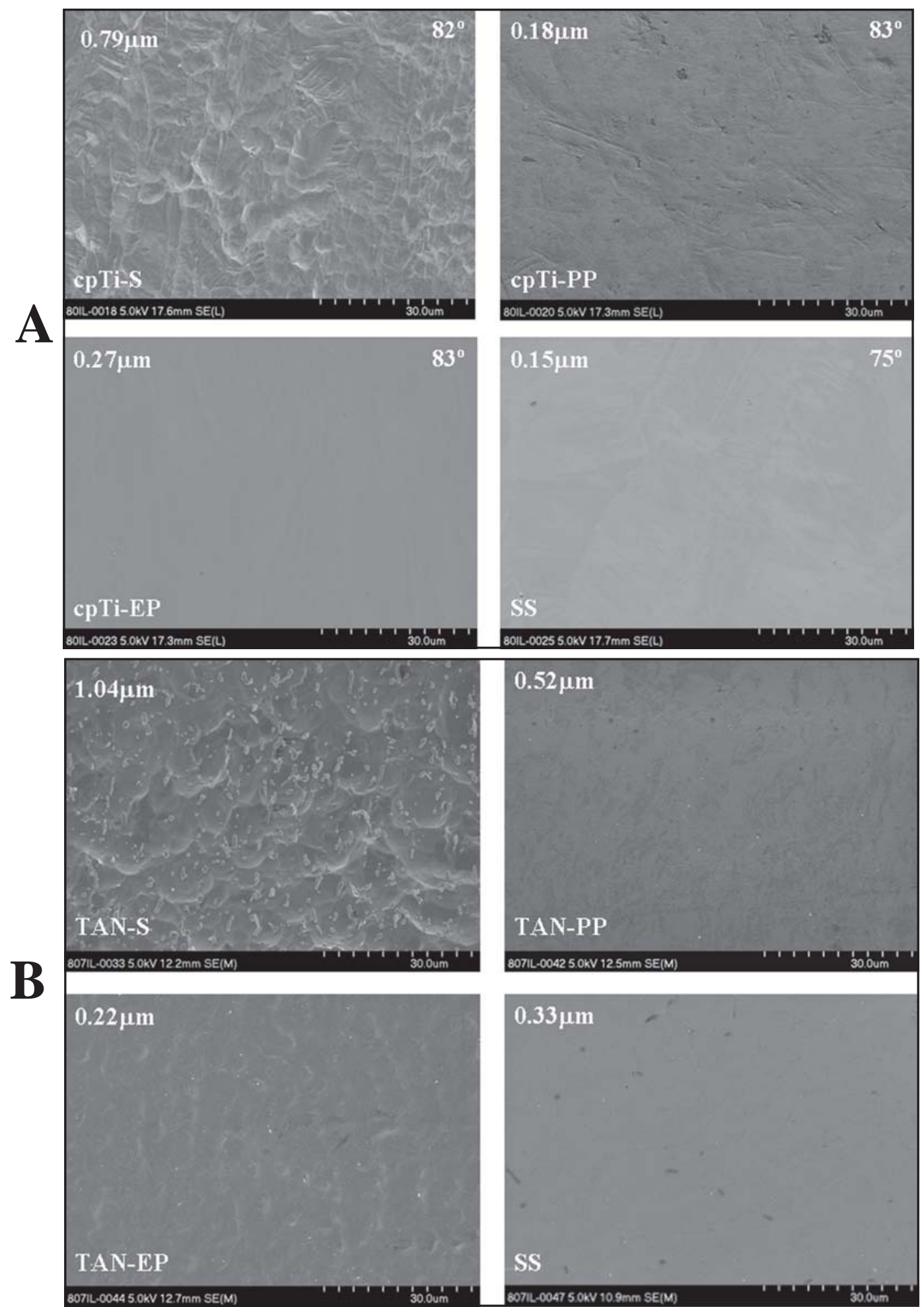

Fig. 1. Surface characterization results of (A) cpTi plates, and (B) TAN screws used in the study. Surface polishing effectively reduces the average roughness ( $\mathrm{Ra}$; inset, displayed in micrometers) associated with the commercially available micro-rough surfaces (TAN-S). Both electropolishing (cpTi-EP, TAN-EP) and paste polishing (cpTi-PP, TAN-PP) compare favorably to the orthopedic smooth surface of SS. Electropolishing of cpTi (cpTi-EP) produces an extremely smooth surface, akin to that of SS. Paste polishing cpTi (cpTi-PP) leaves some mechanical abrasions but the overall surface is free from the micro-discontinuities evident for standard cpTi. Neither polishing method appears to alter the hydrophilicity of the LCP's, as all cpTi plates were found to have similar degrees of wettability (inset, in degrees). Due to the small dimensions of the screws, contact angle was not performed on these samples. The characteristic 'micro-spiked' appearance of standard micro-rough TAN (TAN-S) is considerably influenced by both polishing techniques. Electropolishing (TAN-EP) markedly reduces the micro-roughness of TAN-S, creating a smooth surface with visible waviness. Paste polishing (TAN-PP) appears particularly suited for creating a smooth surface, void of waviness. The efficacy of this method has resulted in the beta phase particles, characteristic of clinically available TAN (TAN-S), becoming almost indistinguishable. 
Table 1. X-ray photoelectron spectroscopy results for the plates and screws used in this study.

\begin{tabular}{|cccccccccc|}
\hline Phtes & Ti2p & O ls & Al $\mathbf{2} p$ & Nb 3d & Cls & Ca2p & Nls & NaKLL & P2p \\
\hline cpTi-S & 18.8 & 55.4 & 1.7 & 0.0 & 21.0 & 0.2 & 0.3 & 0.4 & 2.5 \\
cpTi-PP & 15.5 & 48.8 & 0.0 & 0.0 & 31.2 & 0.2 & 0.6 & 0.3 & 2.2 \\
cpTi-EP & 17.1 & 42.6 & 0.0 & 0.0 & 38.7 & 0.2 & 1.2 & 1.2 & 0.0 \\
\hline Strews & & & & & & & & & \\
\hline TAN-S & 10.6 & 38.8 & 1.3 & 0.2 & 47.5 & 0.2 & 0.0 & 0.6 & 1.3 \\
TAN-PP & 12.2 & 42.8 & 1.7 & 0.3 & 40.7 & 0.2 & 0.3 & 0.6 & 1.7 \\
TAN-EP & 13.5 & 43.9 & 2.2 & 0.8 & 39.4 & 0.1 & 0.0 & 1.4 & 0.0 \\
\hline
\end{tabular}

No major differences in the chemical composition of the surfaces were noted for polished plates or screws, compared to their standard micro-rough counterparts. Plates: cpTi-S-standard micro-rough cpTi; cpTi-EP-electropolished cpTi; cpTi-PP-paste polished cpTi. Screws: TAN-S-standard micro-rough TAN; TAN-EP-electropolished TAN; TAN-PP-paste polished TAN.

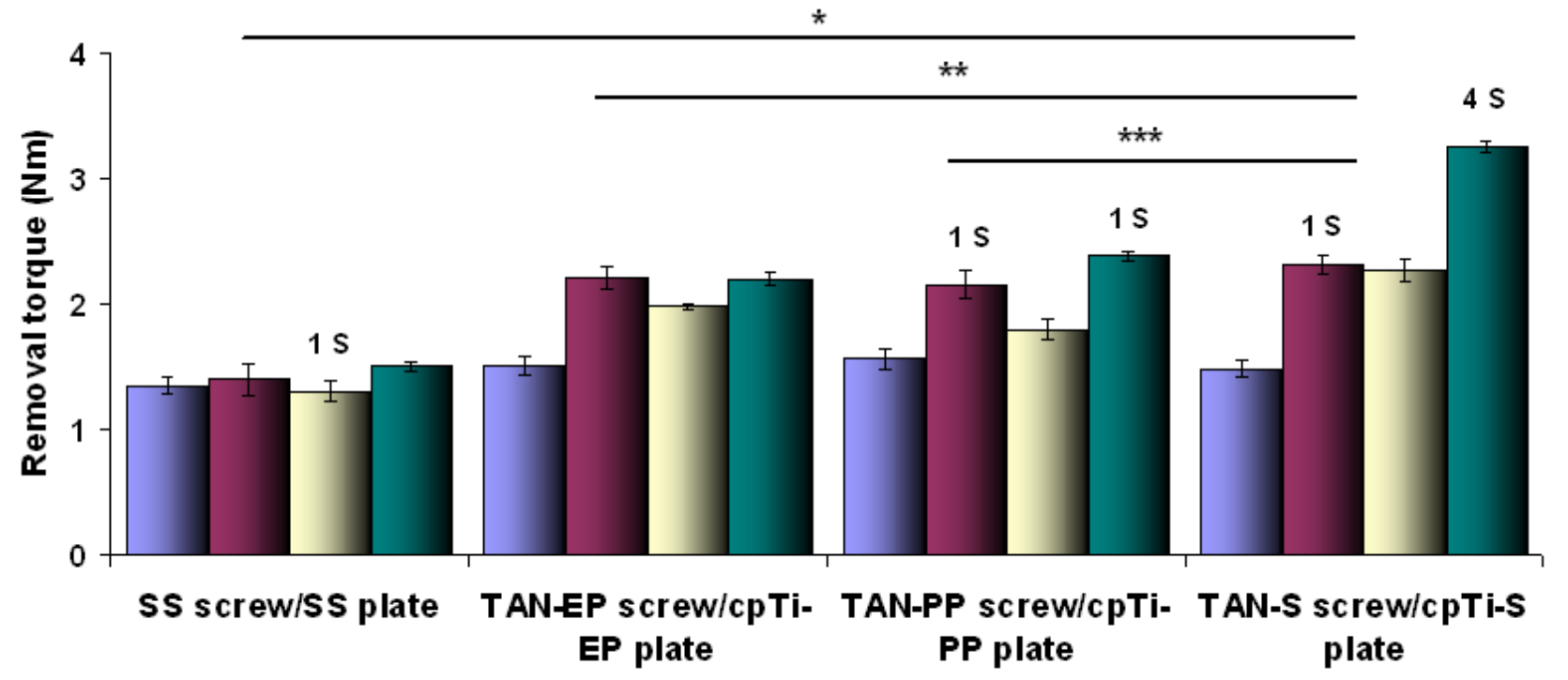

$\square$ Initial $\square 6 \mathrm{mo} \square 12 \mathrm{mo} \square 18 \mathrm{mo}$

Fig. 2. Mean removal torque for cortical screws after initial implantation, and 6, 12 and 18 months' post-implantation. Results indicate that surface micro-topography significantly influences screw removal. Electropolished TAN (TANEP) screws $(* * * p=0.001)$, paste polished TAN (TAN-PP) screws $(* * p=0.010)$ and stainless steel (Ss) screws $\left({ }^{*} p<0.001\right)$ were significantly easier to remove compared to standard micro-rough TAN (TAN-S) screws. This difference was also highlighted by the fact that TAN-S screws had a higher amount of screws that stripped upon attempting to remove (number of stripped screws denoted by ' $\mathrm{S}$ ' above respective construct). Lines with $p$ values shown represent observed significances between surface types.

and Buprenorphin (Temgesic $\AA ; 0.1 \mathrm{mg} / \mathrm{kg}$, i.m., q8h) for 3 days. Sheep were euthanized at 6,12 and 18 months post-operatively with an overdose of Pentobarbital (Vetanarcol ${ }^{\circledR}$ ). Subsequently, the tibiae were harvested and major soft tissues removed. Tibiae containing the plates and screws were wrapped in saline soaked gauze to prevent desiccation.

\section{Torque measurements}

In surgery after insertion of the screws to $1.5 \mathrm{Nm}$ the screws were immediately loosened with a torque measuring screwdriver (Advanced Force Gauge, Mecmesin, Slinfold, West Sussex, UK). This procedure measured the frictional effect of the surface topographies being investigated before the additional effect of bone-on-growth (Fig. 2). Measurement of removal torque force was also performed after euthanasia at 6,12 and 18 months implantation on three of the screws from each plate using an instrumented screwdriver (Advanced Force Gauge, Mecmesin). Tissue adhering to the screw head was removed to enable adequate screwdriver contact. The same operator was used to perform all of the torque removal tests.

\section{Histomorphometric analysis}

Histomorphometric analyses were performed on the one intact screw from each plate to assess the amount of bone contact. A segment of the plate was cut with the screw attached. The segments were fixed in methanol, dehydrated in ethanol and then embedded with methylmethacrylate. Sections were cut with an annular saw then ground (Exact Micro Grinding System, Norderstedt, Germany) and polished to a thickness of approximately $100 \mu \mathrm{m}$ and stained with Giemsa and Eosin. Light microscopic images were taken using a Zeiss Axioplan microscope (Carl Zeiss, 

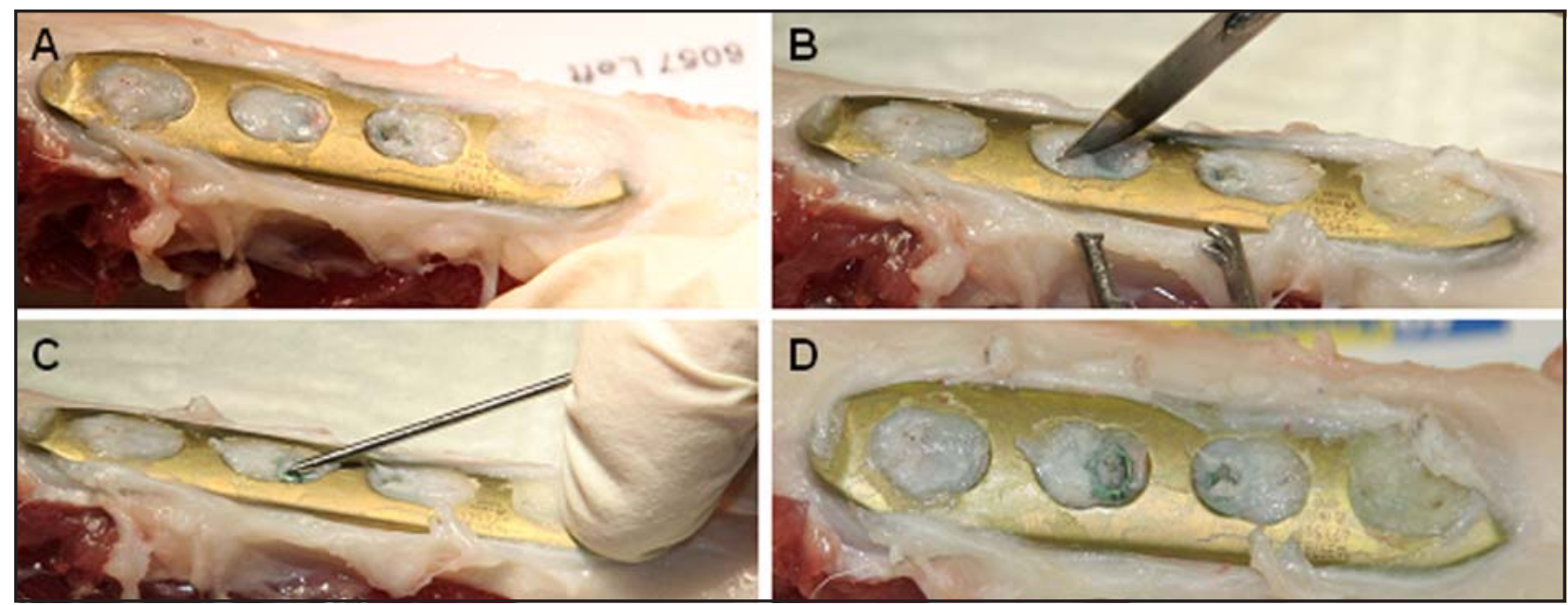

Fig. 3. Attempted clearing of soft tissue and bone from the plate holes of the standard micro-rough cpTi LCP, containing the standard micro-rough TAN screws prior to torque removal tests. After removal of adherent soft tissue, the presence of bone within the plate holes is evident (A). At first, it was attempted to displace this hard tissue with a scalpel (B). Further attempts to breach the bone with a K-wire to expose the screw head proved unsuccessful (C). As the screw heads generally remained unexposed at this point (D), samples would be transferred to a vice, and the bone was subsequently removed using a hammer and chisel. This resulted in the operational time of tissue removal been dramatically increased to approximately 4 times that noted for polished samples.

Göttingen, Germany). Using the computer software package AxioVision 3.1. (Carl Zeiss Vision GmbH, Germany) the direct bone-implant contact was measured for each screw.

\section{Statistical analyses}

Statistical evaluations were made using SPSS for Windows Version 14.0 (SPSS Inc, Chicago, IL, USA). As all 4 materials were implanted within the same sheep, the influence of the material as a within-subject factor and time as a between-subject factor upon removal torque (statistical power $=1.000)$ and percentage bone contact (statistical power $=0.906$ ) was analyzed by repeatedmeasures ANOVA with Bonferroni post hoc test for individual group comparisons. To increase the statistical power, data for each time point for a given surface type was collected; therefore $p$ values within the text represent significant observations for the given surface pertaining to the factor tested. Values less than or equal to 0.05 were deemed significant.

\section{Results}

\section{Surface characterisation}

Both the roughness average and morphological roughness appearance of the plate (Fig. 1A) and screw (Fig. 1B) surfaces were greatly reduced by polishing. Results from contact angle measurements of the plates indicate that surface polishing did not alter the wettability of the LCPs to any considerable extent, as all LCPs had contact angles of approximately $83^{\circ}$. Analysis of the surface chemistry using XPS (Table 1) showed that neither polishing method (cpTi-EP /TAN-EP; cpTi-PP/TAN-PP) affected the final surface chemical properties from the positive controls (cpTi-S /TAN-S). As expected, spectra for cpTi plates and
TAN screws are dominated by Ti and $\mathrm{O}$ due to the naturally occurring titanium oxide layer. For TAN samples, in addition to $\mathrm{Ti}$ and $\mathrm{O}$, the alloying elements ( $\mathrm{Al}$ and $\mathrm{Nb}$ ) were also present.

\section{Torque removal measurements}

Results indicate that the frictional effect for screw insertion and immediate extraction was similar between materials (Fig. 2). Surface polishing as a material treatment had a significant influence on the torque required for screw removal (Fig. 2). It was observed that TAN-EP $(p=0.001)$, TAN-PP $(p=0.010)$ and SS screws $(p<0.001)$ were significantly easier to remove compared to TAN-S screws. Additionally, we found that SS screws were significantly easier to remove compared to TAN-EP $(p=0.002)$ and TAN-PP $(p<0.001)$ screws. However, no statistical difference was noted between TAN-EP and TAN-PP $(p=1.000)$. Moreover, no statistical difference was observed regarding the time of implantation and its effect on torque removal $(p=0.07)$. No statistical difference was noted between 6 and $12(p=1.000), 6$ and $18(p=0.247)$ or 12 and $18(p=0.090)$ month results for torque removal related to time. However, it was noted that with increasing time TAN-S screws became more difficult to remove and resulted in increased screw stripping compared to polished screws (Fig. 2). It should be noted that the unpolished screws that stripped after 18 months were unable to be removed.

\section{Ex vivo observations}

After removal of adhering soft tissues a clear difference in tissue attachment between constructs was evident. Fig. 3 highlights the difficultly incurred in removal of bone from the standard micro-rough systems. After removal of adherent soft tissue the presence of bone within the plate holes was evident. We attempted to displace this with a K- 

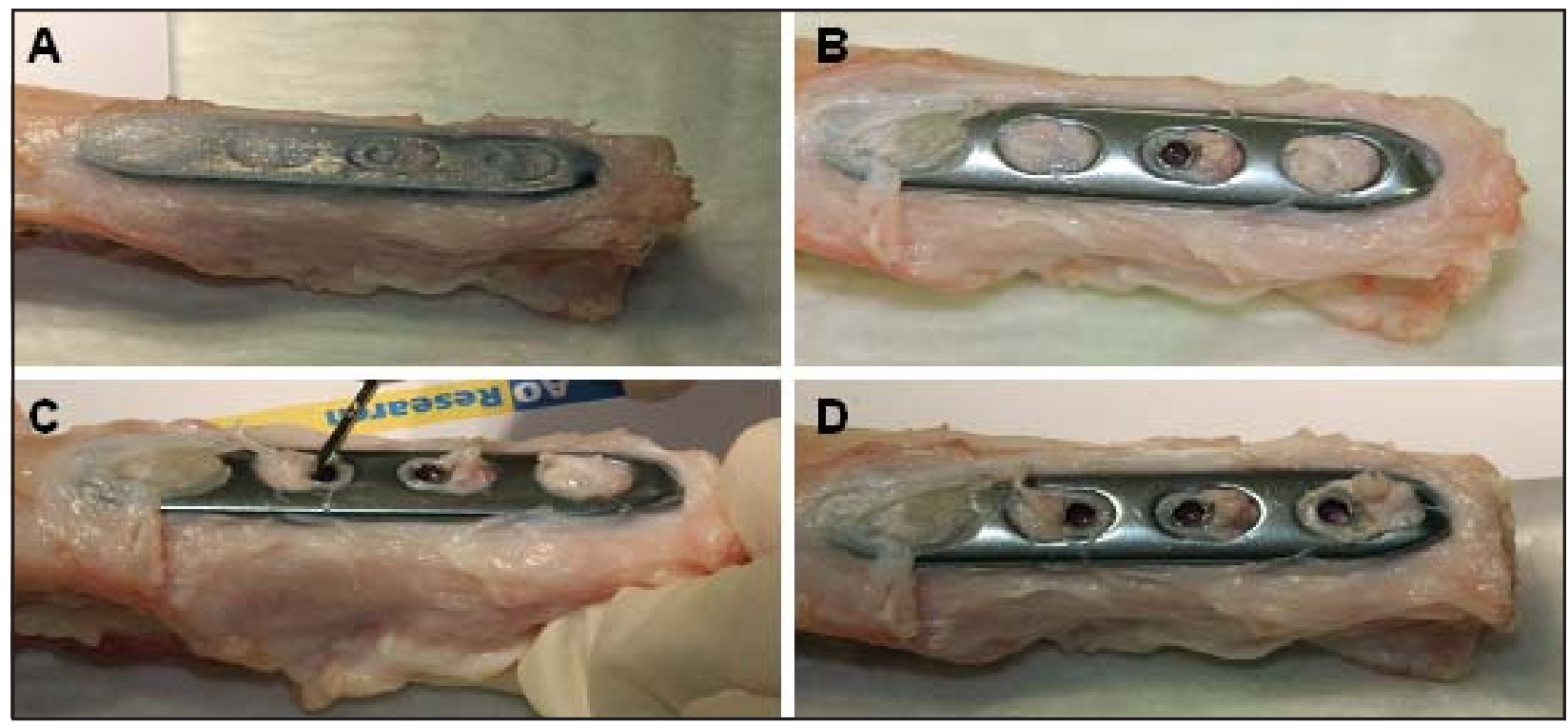

Fig. 4. A thin layer of fibrous tissue was generally observed to be adhered to polished constructs (A). This layer was easily removed to expose the underlying samples without damage to the plates (B). Soft tissue was observed within the plate holes of the polished samples which was easily displaced by a K-wire (C) to expose the screw heads for subsequent removal torque analysis (D). After 18 months, bone was sometimes noted within the plate hole of some of the polished constructs; however, this was infrequent, and easily removed. The operational time for oft tissue removal was almost instantaneous, and resulted in a total operational time of approximately 5 minutes, to the point were adequate access to the screw holes was achievable.

wire and a scalpel without success. As the screw heads generally remained unexposed at this point, samples were transferred to a vice, and the bone was removed using a hammer and chisel. This bone became increasingly difficult to remove, dramatically increasing the time for removing excess tissue in order to expose the screw holes to four times that noted for polished samples (20 minutes compared to 5 minutes for polished samples). Polished samples on the other hand proved considerably easier (Fig. 4). After removal of the adhering soft tissue the presence of soft tissue within the plate holes and screw heads was evident. In contrast to micro-rough constructs the tissue within the screw head was easily removed with a K-wire. After 18 months implantation some bone in-growth was noted in some of the polished plate holes however, this was infrequent and did not pose the same difficulty for removal as the micro-rough systems. The total time taken to expose the screw heads of the polished systems generally took less than 5 minutes.

\section{Histomorphometric analysis}

In relation to surface dependent response, surface microtopography was found to significantly affect the percentage of bone contact to the screws (Fig. 5). While this decrease in bone contact was found to be significant for TAN-PP screws $(p<0.001)$ bone contact on TAN-EP screws was not found to differ significantly $(p=0.066)$ compared to TAN-S screws. Nevertheless, no significant difference in the percentage of bone contact was observed between the polished variants $(p=0.752)$. As expected, SS screws had significantly less bone contact compared to TAN-S screws $(p<0.001)$ however, TAN-EP $(p<0.001)$ and TAN-PP $(p=0.007)$ screws also had significantly higher percentage of bone contact compared to SS screws. In terms of the influence of time on the percentage of bone contact no significant differences between 6 and $12(p=0.567), 6$ and $18(p=0.339)$ or 12 and $18(p=1.000)$ months was observed for all material combinations. No correlation was found between torque removal and percentage of bone contact for SS $(p=0.272)$, TAN-EP $(p=0.172)$ or TAN-PP $(p=0.402)$ screws. However, TAN-S screws did show a correlation for removal torque and percentage of bone contact $(p=0.026)$.

These observations were supported histologically (Fig. $6)$. For TAN-S screws, the high frequency of bone apposition to the implant is evident over time. TAN-EP and TAN-PP screws, while also displaying a high degree of bone contact, had a higher incident of fibrous tissue compared to TAN-S screws. SS screws in contrast to all other samples displayed the persistent presence of a continuous, very thin, fibrous layer around the screw, separating it from adjacent hard tissue.

\section{Discussion}

The benefits of osseointegration for long-term functional success of internal fixation devices such as spine cages and hip prostheses are indisputable. Indeed extensive studies have focused on enhancing osseointegration through surface microtopography manipulation, which is a major determining factor for osseointegration (Albreksston and Hansson, 1986; Pearce et al., 2008; Hayes et al., 2009a). However, we postulate that direct, strong osseointegration provided by 'standard' microrough IF is not necessary for fracture fixation implant 


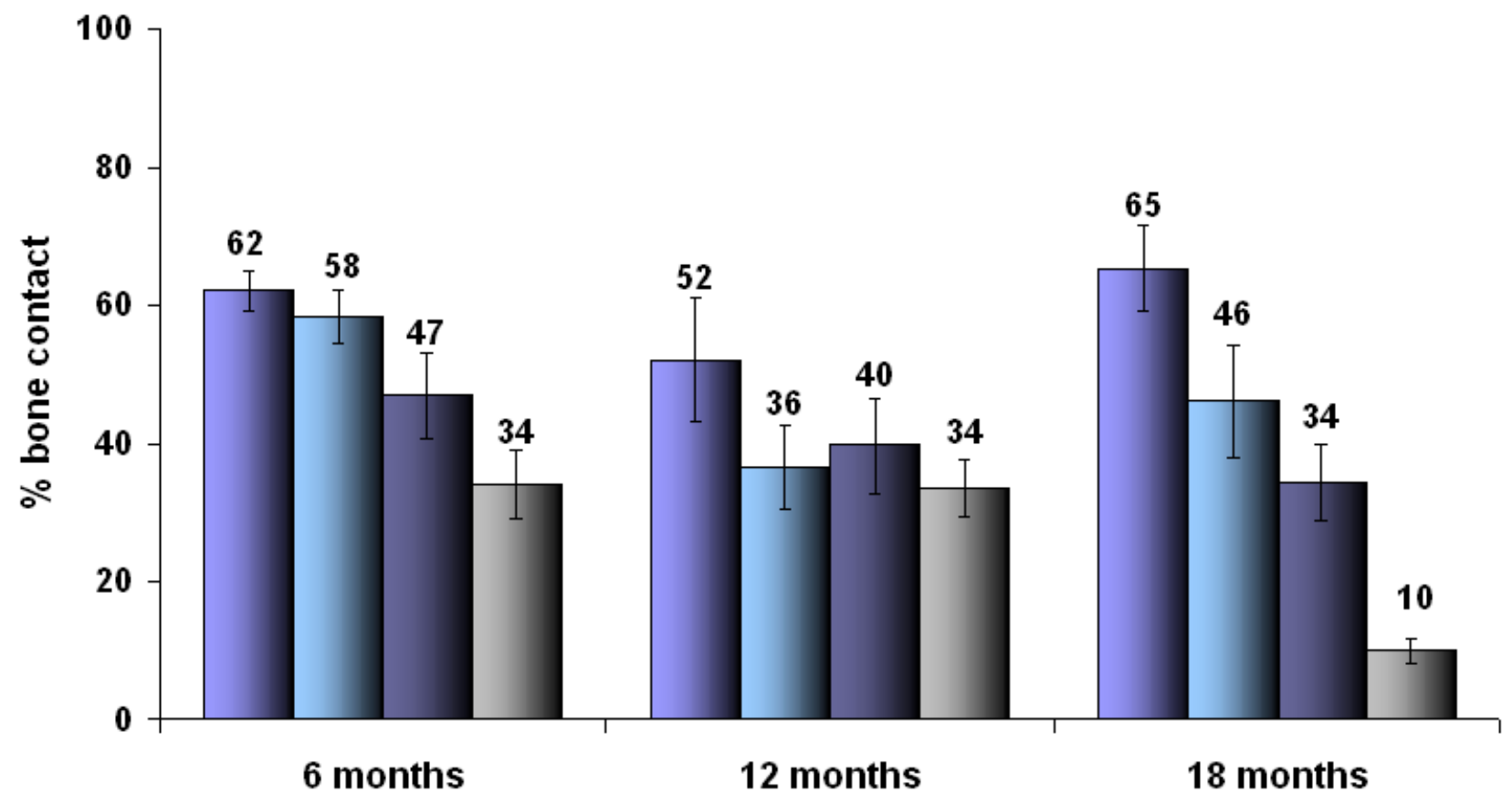

$\square$ Standard microrough TAN $\square$ Electropolished TAN $\square$ Paste polished TAN $\square$ Stainless steel

Fig. 5. Percentage of bone contact for TAN-EP, TAN-PP, TAN-S and SS screws after 6, 12 and 18 months implantation, \pm S.E.M. SS screws had significantly less bone contact compared to TAN-S $(p<0.001)$, TAN-EP $(p<0.001)$ and TAN-PP $(p=0.007)$ screws. However, TAN-PP screws significantly influenced the quantity of bone contact, compared to TAN-S $(p<0.001)$, but this was not observed for TAN-EP screws $(p=0.066)$. Furthermore, no significant difference in bone contact was noted for polished TAN screws $(p=0.752)$. No correlation was found between torque removal and percentage of bone contact for Ss $(p=0.272)$, TAN-EP $(p=0.172)$ or TAN-PP $(p=0.402)$ screws. However, TAN$\mathrm{S}$ screws did show a correlation for removal torque and percentage of bone contact $(p=0.026)$.

stability in temporary devices and in fact reduction in the strength of this bone-material interaction through polishing can positively influence removal related morbidity. We do not suggest that polishing of IFs should be used as the standard of care for all fracture fixation applications. In pediatric and trauma fracture fixation temporary implantation of devices may be preferable and polished IFs would greatly reduce removal related complications within these patients.

In this study, we show that surface polishing of internal fixator materials significantly eases screw removal from a locked plate system. These results support earlier observations from our laboratory that showed that polishing of conventional bone screws made of cpTi and TAN significantly reduces their removal from both cortical and cancellous sheep bone after 6,12 and 18 weeks (Pearce et al., 2008). More recently, we have also shown that polishing of TAN intramedullary nails can significantly reduce the pullout force required for removal after 18 months implantation within sheep. This result was associated with the formation of a fibro-osseous interface, compared to direct bone bonding observed for standard microrough TAN nails (Hayes et al., 2009a). Nevertheless, no incidences of nail instability were observed. In this study, after 12 months in vivo a small decrease in removal torque was observed for all surface combinations. A similar trend for decreased removal torque with increasing implantation has been previously noted (Eulenberger and Steinemann 1990; Pearce et al., 2008) although both these studies did encompass shorter implantation periods than in this study. However, the slight decrease in removal torque is postulated to represent a period of bone remodeling, hence the lower extraction force required for screw removal at this time. Since there was no indication of implant instability or screw loosening for any of the combinations studied here or in other studies (Eulenberger and Steinemann, 1990; Pearce et al., 2008) it would appear that, at least in these models, the slight decrease in extraction torque has minimal impact on the interfacial stability of the screws, or adjacent tissue healing/ remodeling.

Histomorphometric results indicate that paste polished TAN (TAN-PP) screws produce a significantly lower percentage of direct bone contact compared to standard microrough TAN (TAN-S) screws while electropolished TAN (TAN-EP) screws did not, although they did show a trend for lower bone contact. This is not a surprising observation; however, as clearly the morphology of the TAN-PP screws had a lower surface roughness than TANEP, and morphologically was smoother. For both polished screw types no statistical correlation was found between the quantity of direct bone contact and torque removal. Therefore, we suggest that the strength of bone attachment to polished screws is less compared to TAN-S and that this is contributed to the reduced removal torques. This is supported by the fact that polished screws had less stripping compared to micro-rough TAN screws. This may in part be explained by in vitro observations recently made by 


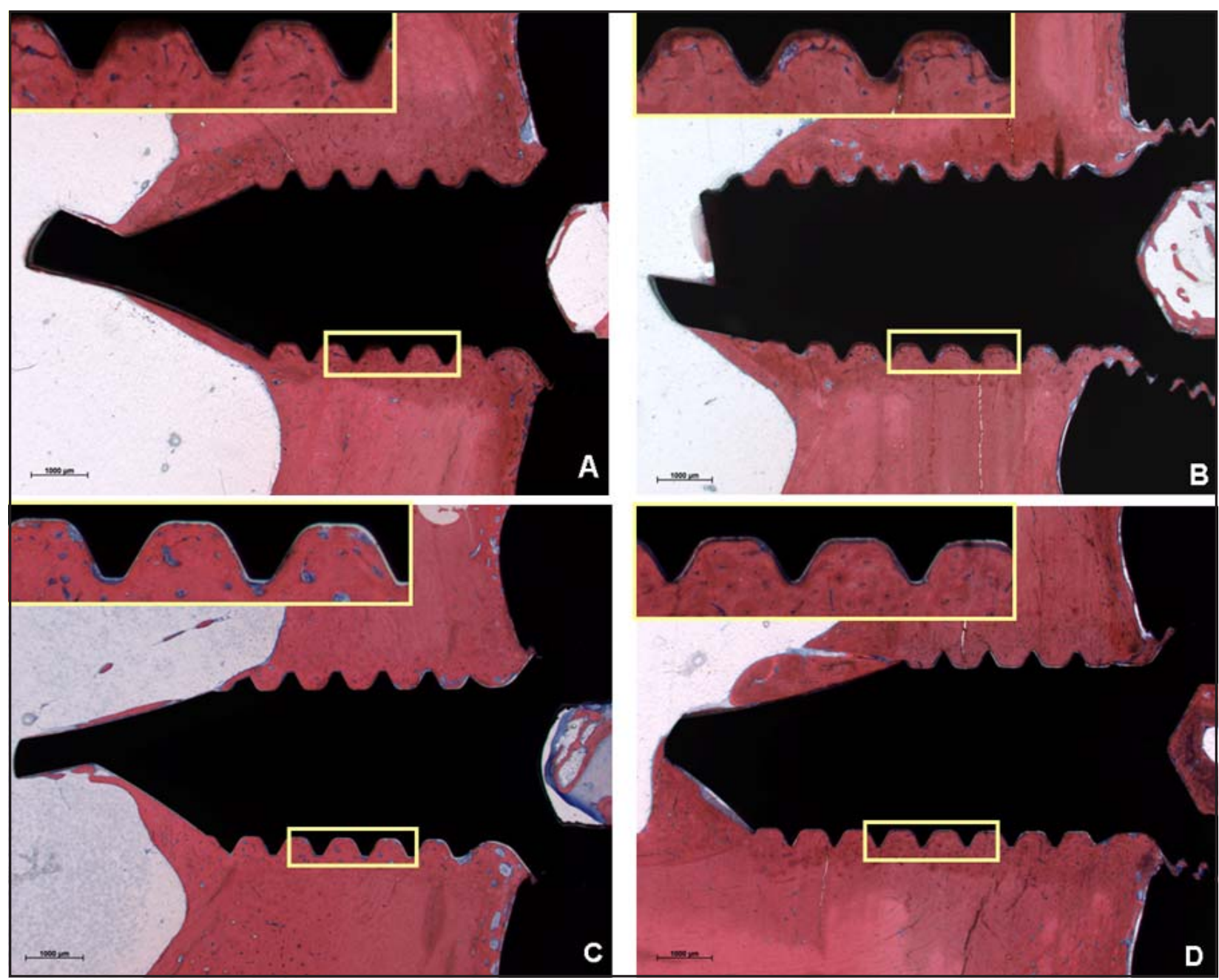

Fig. 6. Representative histology images of (A) TAN-S, (B) TAN-EP, (C) TAN-PP and (D) SS screws after 18 months implantation. Sections are stained with Giemsa-Eosin for detection of bone (pink) and soft tissue (blue). TAN-S screws displayed high bone contact, with small intermittent areas of soft tissue (fig 6A, inset). TAN-EP and TAN-PP screws had a similar tissue reaction as seen for TAN-S. High bone contact was noted, with some areas displaying the presence of a thin, fibrous layer. SS screws had little or no bone contact after 18 months implantation. Instead, a thin, continuous, fibrous layer surrounded the screw (fig.6D, inset).

our group that indicate that for similar material and surface preparations polished samples had a slower rate of terminal osteoblast differentiation compared to standard samples (Hayes et al., 2009b).

While a statistical correlation was noted for cpTi-S/ TAN-S systems for the percentage of bone contact and removal torque of the screws, this correlation was not observed for any other sample combination, including SS. Consequently, as mentioned previously, our results indicate that the strength of bone attachment rather than the amount of bone at the surface is the key contributor for ease of removal. This reduction in percentage of bone contact to polished TAN screws does not appear to negatively influence the interfacial tissue-implant relationship as bone apposition to the implant was not prohibited nor did we encounter any incident of screw/implant instability over the course of the 18-month implantation period.

Histologically, bone was the principal tissue type within the thread of polished screws with only small intermittent areas of fibrous tissue being evident. If however, the more challenging fracture model was to induce a fibro-osseous interface with the screw, it is likely that the interfacial strength of this interface would still be sufficient to maintain implant stability and allow for uninterrupted healing to occur. Evidence in support of this statement is provided by the success of stainless steel devices that are implanted without incident world-wide annually $(\sim 700,000$ trauma SS pieces in the U.S alone), despite the lack of evidence of direct osseointegration to steel IFs. Histological and removal torque data for SS screws from this study also support this hypothesis as direct osseointegration to SS screws was prevented by the formation of a thin, continuous fibrous tissue layer. These findings are also supported by earlier studies from our laboratory (Pearce et al., 2008; Hayes et al., 2009a) and others (Albreksston \& Hansson, 1986). Furthermore, we have shown here that SS screws had significantly lower percentage of bone contact compared to both microrough and polished TAN screws. Even so, no issues of screw instability were noted for SS screws. A similar outcome has been observed for conventional bone screws (Albreksston and Hansson, 1986; Pearce et al., 2008) and 
intramedullary nails (Hayes et al., 2009a), without any compromise in implant stability being noted. Therefore, within the context of this study it appears that despite the reduction in bone contact to polished screws, interfacial strength appears to be sufficient to retain implant stability. Moreover, the clinical success of SS implants in the absence of direct osseointegration would suggest that if a further reduction in interfacial strength becomes evident for polished screws within a healing environment of a fracture model, as long as this remained within a range of SS then implant instability is unlikely. This hypothesis would, however, have to be tested within a fracture model to be definitive.

Increased surgery time is an inevitable complication of difficulty in removing an IF with many reports suggesting occurrence in as many as $22-66 \%$ of patients (Swiontkowski et al., 1983; Alzahrani et al., 2003; Ilchmann and Parsch, 2006). Alzahrani and colleagues (2003) reported a significant increase in the surgical time for pediatric patients that underwent device removal, which due to the lengthy implantation duration and abundant callus formation that directly result in increasing the surgical time and blood loss. In this study we have shown that polishing can positively influence this outcome by reducing the time required for device extraction. Our previous studies have already highlighted the advantage of polishing commercially available IF plates for tendon and muscle gliding over implants to allow free movement to reduce the formation of restrictive adhesions and consequently irritation and inflammation of adjacent tissues (Richards et al., 2000; Welton, 2007). It may be suggested that the increased time observed for clearing of 'standard' systems may only apply to the method used in this study since dedicated removal kits are available when such problems are encountered. The authors agree that such kits would improve this time pressure although these sets may also introduce heat problems and mass debris contamination and do not always speed up the removal process. Our ex vivo observations suggest that the occurrence of problematic screws and plates would be markedly reduced by prevention through polishing the implants thereby reducing the need of introducing these sets.

As mentioned throughout, the major limitation of this study is the inclusion of a non-fracture model. While the extent of trauma is not equivalent to that caused by fracture, trauma to the sheep was initiated through the surgical wound and insertion of the plate and screws. The authors recognize the value of reproducing the in vivo physiological niche associated with a fracture model. However, the nonfracture model allowed for conclusions to be drawn specifically related to material-surface dependent responses, which was the point of this initial study, rather than biological responses based on variations in healing that would exist if a fracture model was included. The nonfracture model also allowed a bilateral comparison removing the variance between the groups. Nevertheless, the authors concede that while this study provides a strong foundation for advocating surface polishing fore reducing removal related morbidity, this technology needs to be assessed under the more challenging circumstance of fracture healing for the potential of surface polishing to be found fully comprehensive. A comparative model would, however, require a much greater number of sheep to allow for the anatomical and metabolic variances between the sheep.

\section{Conclusion}

The significant reduction in the extraction force required for removal of polished screws as well as the reduction in time required for tissue removal from polished plates/ screws should directly reduce the surgical time associated with implant removal. Thus polishing of titanium or titanium alloy fracture fixation implants may not only improve the economic burden associated with surgical procedures, but also the surgical complications experienced by the patient which are both principal deciding factors for implant removal. Future studies aim to assess the potential of this technology in the next level of complication, a fracture model.

\section{References}

Albreksston T, Hansson HA (1986) An ultrastructural characterisation of the interface between bone and sputtered titanium or steel surfaces. Biomaterials 7: 201205

Alzahrani AG, Behairy YM, Alhossan MH, Arab FS, Alammari AA (2003) Removal of internal fixation devices in pediatric patients. Saudi Med J 24: 254-255

Babst R, DeSimoni D, Dieter U, Frigg R, Gächter A, Gautier E, Helfet DL, Korner J, Kuster S, Lill H, Linke B, Lobenhoffer P, Müller M, Perren S, Rommens PM, Schneider E, Sommer C, Stachowiak G, Staubli E, Wagner M (2003) Locking compression Plate-LCP-a new AO principle. Injury Supplement 2 vol. 34.

Bhatt V, Chhabra P, Dover MS (2005) Removal of miniplates in maxillofacial surgery: a follow-up study. J Oral Maxillofac Surg 63: 756-760.

Bostman O, Pilhajamaki H (1996) Routine implant removal after fracture surgery: A potentially reducible consumer of hospital resources in trauma units. J Trauma 41: $846-849$.

Boyan BD, Bonewald LF, Paschalis EP, Lohmann CH, Rosser J, Cochran DL, Dean DD, Schwartz Z, Boskey AL (2002). Osteoblast-mediated mineral deposition in culture is dependent on surface microtopography. Calcif Tissue Int 71: 519-529.

Busam ML, Esther RJ, Obremskey WT (2006) Hardware removal: Indications and expectations. J Am Acad Orthop Surg 14: 113-120.

Case CP, Langkamer VG, James C, Palmer MR, Kemp AJ, Heap PF, Solomon L (1994) Widespread dissemination of metal debris from implants. J Bone Joint Surg Br 76: 701-712.

Emel E, Güzey D, Seyithanolu H, Sel B, Alata_ I (2007). Delayed infection 6 years after spinal instrumentation: a case report. Turk Neurosurg 17: 16-20. 
Eulenberger J, Steinemann SG (1990). Lösemomente an Kleinschrauben aus Stahl und Titan mit unterschiedlichen Oberflächen.(Removal torques in small steel and titanium screws with different surfaces.) Unfallchirurg 93:96-99

FerrisDM, Moodie GD, Dimond PM, Gioranni CW, Ehrlich MG, Valentini RF (1999) RGD-coated titanium implants stimulate increased bone formation in vivo. Biomaterials 20: 2323-2331.

Georgiadis GM, Gove NK, Smith AD, Rodway IP (2004) Removal of the less invasive stabilisation system. J Orthop Trauma 18: 562-564.

Hanson B, van der Werken C, Stengel D (2008). Surgeon's beliefs and perceptions about removal of orthopaedic implants. BMC Muscolskel Dis 9: 73

Hayes JS, Vos DI, Hahn J, Pearce SG, Richards RG (2009a) An in vivo evaluation of surface polishing of TAN intramedullary nails for ease of removal. Eur Cells Mat 18: $15-26$.

Hayes JS, Archer CW, Richards RG (2009b) Control of integration at a cellular level: Influence of surface polishing on bony on-growth. Tran $55^{\text {th }}$ Annu Meeting Orthop Res Soc, Las Vegas, NV, USA, poster 481.

Highland TR, LaMont RL (1985) Deep, late infections associated with internal fixation in children. J Pediatr Orthop 5: 59-64.

Ilchmann T, Parsch K (2006) Complications at screw removal in slipped capital femoral epiphysis treated by cannulated titanium screws. Arch Orthop Trauma Surg. 126: $359-363$.

Kahle WK (1994) The case against routine metal removal. J Pediatr Orthop 14: 229-237.

Kasai Y, Iida R, Uchida A (2003) Metal concentrations in the serum and hair of patients with titanium alloy spinal implants. Spine 15: 1320-1326.

Keegan GM, Learmonth ID, Case CP (2007) Orthopaedic metals and their potential toxicity in the arthroplasty patient. J Bone J Surg 89: 567-573.

Kim WY, Zenios M, Kumar A, Abdulkadir U (2005) The removal of forearm plates in children. Injury, Int $\mathrm{J}$ Care Injured 36: 1427-1430.

Mosbah MR, Oloyede D, Koppel DA, Moos KF, Stenhouse D (2003) Miniplate removal in trauma and orthognathic surgery-a retrospective study. Int J Oral Maxillofac Surg 32: 148-151.
Nazzal A, Lozano-Calderón S, Jupiter JB, Rosenzweig JS, Randolph MA, Lee SG (2006) A histologic analysis of the effects of stainless steel and titanium implants adjacent to tendons: an experimental rabbit study. J Hand Surg [Am] 31: 1123-1130.

Pearce AI, Pearce SG, Schwieger K, Milz S, Schneider E, Archer CW, Richards RG (2008) Effect of surface topography on removal of cortical bone screws in a novel sheep model. J Orthop Res 26: 1377-1383.

Peterson HA (2005) Metallic implant removal in children. J Pediatr Orthop 25: 107-115.

Qu, J, Chehroudi, B, Brunette, DM (1996) The use of micromachined surfaces to investigate the cell behavioural factors essential to osseonintegration. Oral Dis 2:102-115.

Rallis G, Mourouzis C, Papakosta V, Papanastasiou G, Zachariades N (2006) Reasons for miniplate removal following maxillofacial trauma: A 4 year study. J CranioMaxillo Surg 34: 435-439.

Richards RG, Persson A, Gasser B, Wieling R (2000) Influence of surface microtopography on formation of capsules: An in vivo study of stainless steel implants in rabbits. Trans $10^{\text {th }}$ Annu Conf Eur Orthop Res Soc, Wiesbaden. Trans Vol 10: 0-136

Rosson JW, Shearer JR (1991) Refracture after removal of plates from the forearm. J Bone J Surg 73: 415-417. Seipel RC, Schmeling GJ, Daley R (2001) Migration of a $\mathrm{K}$-wire from the distal radius to the heart. Am J Orthop 30: 147-151.

Sommer B, Felix R, Sprecher C, Leunig M, Ganz R, Hofstetter W (2005) Wear particles and surface topographies are modulators of osteoclastogenesis in vitro. J Biomed Mater Res A 72: 67-76.

Summer B, Fink U, Zeller R, Rueff F, Maier S, Roider G, Thomas P (2007) Patch test reactivity to a cobaltchromium-molybdenum alloy and stainless steel in metalallergic patients in correlation to the metal ion release. Contact Dermatitis 57: 35-39.

Swiontkowski MF. (1983 Slipped capital femoral epiphysis: Complications related to internal fixation. Orthopedics 6: 705-712.

Togrul E, Bayram H, Gulsen M, Kalaci A, Ozbarlas S (2005) Fractures of the femoral neck in children: longterm follow up in 62 hip fractures. Injury 36: 123-130.

Welton JA (2007) In vivo evaluation of defined polished surfaces to prevent soft tissue adhesion. Masters Thesis, AO Research Institute, Davos, Switzerland. 